\title{
Serum platelet-derived growth factor-BB levels as a potential biomarker in assessing the metabolic activity of lesions in alveolar echinococcosis patients
}

\section{ying ke}

Xinjiang Medical University Affiliated First Hospital https://orcid.org/0000-0003-1207-4480

\section{Xiaojuan Bi}

Xinjiang Medical University Affiliated First Hospital

Ning Yang

Xinjiang Medical University Affiliated First Hospital

Xiaohong Li

Xinjiang Medical University Affiliated First Hospital

\section{Wenmei Ma}

Xinjiang Medical University Affiliated First Hospital

Hui Liu

Xinjiang Medical University Affiliated First Hospital

Hui Wang

Xinjiang Medical University Affiliated First Hospital

\section{Liang Li}

Xinjiang Medical University Affiliated First Hospital

\section{Cheng Li}

Xinjiang Medical University Affiliated First Hospital

\section{Yongde Qin}

Xinjiang Medical University Affiliated First Hospital

\section{Tuerganaili Aji}

Xinjiang Medical University Affiliated First Hospital

\section{Yingmei Shao}

Xinjiang Medical University Affiliated First Hospital

\section{Guodong Lü}

Xinjiang Medical University Affiliated First Hospital

\section{Renyong Lin ( $\nabla$ renyong_lin@sina.com )}

State Key Laboratory of Pathogenesis, Prevention, and Treatment of Central Asian High Incidence Diseases, Clinical Medical Research Institute, The First Affiliated Hospital of Xinjiang Medical University https://orcid.org/0000-0001-8073-2495 


\section{Research note}

Keywords: Hepatic alveolar echinococcosis, metabolically active alveolar echinococcosis, platelet-derived growth factor-BB

Posted Date: September 11th, 2020

DOl: https://doi.org/10.21203/rs.3.rs-37213/v2

License: (c) (1) This work is licensed under a Creative Commons Attribution 4.0 International License. Read Full License

Version of Record: A version of this preprint was published at Acta Tropica on December 1st, 2021. See the published version at https://doi.org/10.1016/j.actatropica.2021.106290. 


\section{Abstract}

Objective Alveolar echinococcosis (AE) is a chronic disease caused by the larval stage of Echinococcus multilocularis. Assessing the metabolic activity of $A E$ lesions is critical to evaluate disease progression and survey treatment options. There is an urgent need to identify more rapid, convenient, and noninvasive clinical detection methods to substitute the current techniques. Herein, we evaluated the viability of platelet-derived growth factor-B B (PDGF-BB) as a biomarker for detecting the metabolic activity of AE patients. Concentration of serum PDGF-BB homodimers (sPDGF-BB) was assessed via ELISA.

Correlations of PDGF-BB expression levels with clinicopathological features of AE patients were analyzed using SPSS.

Results The concentrations of sPDGF-BB were significantly lower in AE patients ( $p<0.0001)$, particularly in High Metabolically Active AE patients (HMAE) patients $(p<0.05)$. The expression levels of PDGF-BB were significantly higher in close liver tissue (CLT) in AE patients $(p<0.0001)$. We found that metabolically active $A E$ and SPDGF-BB are significantly negatively correlated $(r=-0.624, p=0.0004)$. Besides, the local expression levels of PDGF-BB was positively correlated with metabolic activity, PNM stage, and lesion size. Notably, the sPDGF-BB levels were proposed as a potential biomarker for assessing metabolic activity of $A E$, with $81 \%$ sensitivity and $85.7 \%$ specificity ( $95 \%$ confidence interval, $p=0.003$ ).

\section{Introduction}

Alveolar echinococcosis (AE) is a gradual and invasive form of echinococcosis caused by Echinococcus multilocularis infection that gradually invades the liver. It is considered a significant global public health concern, highly prevalent in Northwest China, Central Asia, Western Europe, and Middle East(1). The lesions of $A E$ damage the hepatic tissue and obstructs bile ducts and vessels(2). This causes severe or even lethal complications(2).

The status of the parasitic lesion in the patient which can be classified as calcified, highly active, and or general is used to determine the degree of disease progression. Therefore, they are important parameters that affect the decision-making process in clinical treatment and can be used to determine the appropriate time to discontinue albendazole therapy $(2,3)$. The F-labeled fluoro-2-deoxyglucose 18 (FFDG), positron emission tomography and computed tomography (PET-CT) techniques have been integrated and currently provide a superior non-invasive tool for assessing the metabolic activity of $\mathrm{AE}$ lesions(4). However, several limitations of PET-CT such as the high cost of the procedure and the need for professionals and reagents implies that there is an urgent need to develop more economical, convenient, and rapid methods for in vivo analysis of the parasitic activity.

Platelet-derived growth factors (PDGFs) are alkaline proteins stored in platelet alpha granules(5). The isoform PDGF-BB participates in the maturity and reconstructing of the vascular network by recruiting parietal cells (pericytes and vascular smooth muscle cells) around new blood vessels (6). Notably, platelets have a central function in hepatic dynamic balance and pathobiology(7). PDGF-BB as one of the 
platelet-related biomarkers, was released from many different cell types, and played pivotal roles in cell proliferation and transformation, chemotaxis, angiogenesis, wound healing, and the regulation of apoptosis(8). Furthermore, PDGF-BB has demonstrated several implications in the field of oncology and other diseases including screening, diagnostic, and prognostic relevance of diseases. (9-11). For instance, higher serum levels of PDGF-BB in hepatocellular carcinoma (HCC) patients undergoing sorafenib treatment are indicators for extended survival benefits (12). Patients with low levels of serum plateletderived growth factor-BB (sPDGF-BB) during the perioperative period of HCC are more likely to exhibit post-operation relapse (13).

In this study, we investigated the expression of PDGF-BB in peripheral and local tissues in AE patients and determine the prognostic value of SPDGF-BB in assessing the metabolic activity of lesions in AE patients.

\section{Methods}

\section{Human Subjects}

A total of 28 AE patients admitted at the First Affiliated Hospital of Xinjiang Medical University from February 2016 to October 2019 were included in the study cohort. Patients were diagnosed with AE following international recommendations(14). The exclusion criteria included: (1) Patients with cystic echinococcosis. (2) Patients with viral hepatitis, autoimmune diseases, and tumors. We grouped $28 \mathrm{AE}$ patients following the WHO Informal Working Group on Echinococcosis PNM ( $P=$ parasitic mass in the liver, $\mathrm{N}=$ involvement of neighboring organs, and $\mathrm{M}=$ metastasis) classification and staging of alveolar echinococcosis. 28 healthy volunteers from the same demographic origin who received annual health examinations were included as the control group. Detailed baseline information for all subjects is summarized in Table S1.The handling of human subjects followed the principles of the Helsinki Declaration and was approved by the Clinical Research Ethics Committee of the First Affiliated Hospital of Xinjiang Medical University. We obtained informed consent from all participants or their legal custody before commencing the study.

\section{Grouping patients based on lesion 'metabolic activity'}

We performed the [18F] Fluorodeoxyglucose positron emission tomography/computed tomography ([18F] FDG PET-CT) scan of the patients immediately after admission or before the operation. The PET/CT acquisitions were conducted 3 hours after 18F-FDG injection(15). The images were analyzed by two experienced nuclear physicians blinded to the clinical, biological, and pathological characteristics of the patients. Subsequently, following the maximum standardized uptake value (SUV MAX ) of [18F]FDG PET-CT, patients were grouped into High Metabolically Active AE (HMAE SUV $\mathrm{MAX}>4.0)$ and Low Metabolically Active AE (LMAE SUV MAX $\leq 4.0$ ) categories(15). The demographic characteristics of subgroups (HMAE vs. LMAE) did not exhibit any significant difference(Table S1).

\section{Sample acquisition}


Blood samples were collected from the subjects on the admission day. Serum was extracted from the samples through centrifugation at $2,500 \times \mathrm{g}$ for 10 minutes and stored at $-80^{\circ} \mathrm{C}$ awaiting further analysis. Besides, a similar procedure was used to process samples from the control subjects.

Sections of the liver tissue were obtained from the $28 \mathrm{AE}$ patients previously underwent surgical resection. One specimen was obtained from a region close to the parasitic lesion including the metacestode (close liver tissue [CLT], about $0.5 \mathrm{~cm}$ from lesion). Another specimen was obtained from the macroscopically normal liver distant from the lesion (distant liver tissue [DLT], at least $2 \mathrm{~cm}$ distant from lesion) as previously described(16).

\section{Quantifying sPDGF-BB}

The quantities of sPDGF-BB were estimated via enzyme-linked immunosorbent assay (ELISA) tests (Quantizing; R\&D Systems, Minneapolis, MN, USA). The concentrations of sPDGF-BB were adjusted to the platelet count levels which were obtained from a complete blood count.

\section{Immunohistochemistry매HC]}

Liver tissue samples were prepared for immunohistochemical analysis. We analyzed the PDGF-BB staining results using IPWIN (version 4.5.0.29 Image-Pro Plus, California, USA). IHC staining was conducted as described previously by Zhang et al(16). Stained sections were visualized by two senior pathologists blinded to each other's results. Briefly, we incubated tissue sections with primary antibodies for mouse PDGF-BB (Abcam, 1:200 Polyclonal) overnight at $4^{\circ} \mathrm{C}$. Subsequent incubation with corresponding secondary antibodies was performed at room temperature for 1 hour in the dark. Immunoreactivity was observed using a horseradish peroxidase-streptavidin detection system (Abcam).

\section{Statistics}

Statistical analyses were performed using the SPSS statistical software package version 20.0 (SPSS, Inc, Chicago, IL, USA) and GraphPad Prism (version 7.0d for MacOS X, USA, GraphPad Software, San Diego, California, USA). Non-parametric data were analyzed via the Mann-Whitney U test, and categorical data were subjected to the Chi-square test. The data of PNM grade was analyed by Spearman correlation. In between the state of "no correlation at all" $(r=0)$ and "perfect correlation" $(r=1)$, interim values of the correlation coefficient are interpreted by convention. Thus, any value $<0.30$ would be poor correlation and a $p$-value $<0.05$ was considered significant(17). The results of PDGF-BB-positive arealLesion volume and PLT were normalized using the following formula(18).

$$
Y=a+\frac{b-a}{X_{\max }-X_{\min }} \cdot\left(X-X_{\min }\right)
$$

We mapped the range of values of a set of data $X$ to an interval, where $Y$ is the mapped value, $X$ max is the maximum value, and $\mathrm{Xmin}$ is the minimum score in this data set. Data that did not conform to the 
normal distribution such as ALT, AST, WBC,TBIL,DBIL and hospitalization days were log-transformed prior to Pearson correlation analysis.

\section{Results}

\section{Low sPDGF-BB concentration in AE patients}

ELISA results indicated significantly low SPDGF-BB levels in AE patients compared to the healthy control subjects $(p<0.0001 ;$ Fig. $1 A)$. However, sPDGF-BB levels in HMAE patients were significantly lower compared to the LMAE patients ( $p<0.01$; Fig. $1 \mathrm{~B})$.

\section{PDGF-BB is highly expressed in CLT from AE patients}

IHC results showed significantly higher expression of PDGF-BB in the CLT compared to DLT (Fig. 2).

\section{Correlation analysis of PDGF-BB status with other clinical variables in AE patients}

In this section, we analyzed different clinical variables to understand the correlation between PDGF-BB expression, lesion activity, and severity of $\mathrm{AE}$. Of note, the levels of SPDGF-BB negatively correlated with lesion metabolic activity $(r=-0.624, p=0.0004)$ which was expressed by $S_{U} V_{\text {MAX }}$, a numerical value that indicated the fluorodeoxyglucose $F 18$ ([18F]-FDG) uptake by the liver lesions in AE patients, the expression of PDGF-BB ( $r=-0.540, p=0.006)$ in the tissues around AE lesions (Fig. 3A;), but not with PNM stages, volume of lesion, number of lesions, inpatient days, and ALT, and AST levels (Table S2). The expressions of PDGF-BB around the lesion were positively correlated with lesion metabolic activity (SUVmax), the PNM stage, and the lesion size but not with other clinical variables (Fig. 3B and Table S2).

Besides, the lesion metabolic activity (SUVmax) was positively correlated with PNM stage $(r=0.53$, $p=0.004)$, lesion volume $(r=0.418, p=0.037)$ and number of lesions $(r=0.481, p=0.01)$ but not with other clinical variables (Fig. S1 and Table S2).

\section{Diagnostic value of serum PDGF-BB levels in predicting the metabolic activities}

The level of SPDGF-BB was assessed as a potential biomarker for assessing metabolic activity, exhibiting $81 \%$ sensitivity and $85.7 \%$ specificity with AUC $>0.8$ ( $95 \%$ confidence interval, $p=0.003$ ) and a cut-off point of $4.66 \mathrm{pg} / 10^{9}$ platelets. Detailed results on the analysis of the receiver operating characteristic (ROC) curves are presented in Fig. S2.

\section{Discussion}

We present the first report indicating a decrease in serum PDGF-BB levels and increase expression of PDGF-BB in local liver tissues from AE patients. Our results showed that AE patients, particularly those with high-activity lesions have significantly lower levels of serum PDGF-BB compared to healthy subjects, 
and a higher expression level of PDGF-BB around the lesion, a bigger lesion size, a worse disease grade compared to those $A E$ patients with low-activity lesions.

Notably, AE patients require lifelong follow-up after surgery and chemotherapy. A previous study reported recurrence cases nearly 20 years post-surgery (19). The lesion progression has been reported to be extremely slow in immunocompetent and early diagnosed AE patients (2). However, prolonged follow-up must be conducted before ruling on the activity of lesions and regression or progression to determine the need for surgery. Currently, [18F]FDG PET/CT is almost the only available noninvasive tool for detecting metabolic activity in AE patients, however, it is scarce and associated with high cost thereby limiting its usage(4). In Africa, for example, many countries have limited access to [18F]FDG PET/CT noninvasive strategy for managing AE patients due to scarce medical resources. In our study, PDGF-BB was found to potentially predict metabolically active lesions with $81 \%$ sensitivity, $85.7 \%$ specificity, and AUC $>0.8$. The techniques used in assessing the PDGF-BB level in serum are more readily available, and affordable compared with PET. Therefore, it can be applied in most regions globally including the developing nations. Many studies have reported that the angiogenic cytokine PDGF-BB is associated with the prognosis and severity of several diseases of the liver such as hepatocellular carcinoma, liver fibrosis, and chronic viral hepatitis. Besides, a previous report indicated that serum levels of PDGF-BB are significantly correlated with the degree of liver damage and fibrosis in chronic hepatitis B (CHB) patients (20). Additionally, serum PDGF-BB levels have been suggested to decrease with increased fibrosis, indicating that the depleted serum PDGF-BB is a potential biomarker for assessing the fibrosis stage in patients with $\mathrm{CHB}(21)$.

Previously, the underlying mechanism associated with reduced PDGF-BB levels in peripheral blood and increased levels of PDGF-BB in the tissue enclosing the lesion has been obscure. We speculated that this phenomenon could be attributed to the high levels of PDGF-BB secreted in the local tissues. Also, the PDGF-BB generated from platelet a-granules responded chemotactically to some chemotactic factor and arrived around the local tissues may cause the relative decrease at the periphery. In addition, several studies have shown that damaged liver exhibits an influx of many platelets $(7,22)$. White blood cells recruit platelets during inflammation which interacts with the hepatic sinusoidal endothelial cells. These cellular interactions can induce the release of a range of up to 300 bioactive proteins (including cytokines, chemokines, growth factors, hemostatic proteins, bioactive lipids, bactericidal agents6, and PDGF-BB)

from platelet a-granules (23). Increasing evidence highlights the role of PDGF-BB at each stage during the progression of liver injury, repair, and fibrosis $(20,21)$.

\section{Conclusion}

These findings suggest that SPDGF-BB could provide a simple, non-invasive, and rapid strategy for monitoring the status of different stages of $A E$.

\section{Limitations}


This pilot study has some limitations that need to be addressed. First, we used a relatively small sample size, therefore, the results should further be validated using larger patient groups. Second, this study did not examine the molecular mechanisms by which SPDGF-BB is eliminated in AE patients, which would be vital in confirming the actual role of PDGF-BB as a biomarker for AE lesion metabolic activity. Third, we did not characterize the molecular events underling SPDGF-BB depletion in AE patients. This could provide novel strategies for monitoring the diagnosis and treatment of $\mathrm{AE}$.

\section{Abbreviations}

AE: Alveolar echinococcosis

PDGF-BB: Platelet-derived growth factor-BB

PDGFsロPlatelet-derived growth factors

HCC: Hepatocellular carcinoma

sPDGF-BB: Serum platelet-derived growth factor-BB

SUV $_{\text {MAX }} \square$ Maximum standardized uptake value

HMAEDHigh Metabolically Active AE

LMAEDLow Metabolically Active AE

CLTaClose liver tissue

DLTaDistant liver tissue

FDG PET-CT: Fluorodeoxyglucose positron emission tomography/computed tomography

IHCDImmunohistochemistry

ELISA: Enzyme-linked immunosorbent assay

ROC: Eceiver operating characteristic

CHB: Chronic hepatitis B

\section{Declarations}

\section{Availability of data and materials}

The datasets used and/or analyzed during the current study are available from the corresponding author on reasonable request. 


\section{Ethics approval and consent to participate}

The study was approved by the Clinical Research Ethics Committee of the First Affiliated Hospital of Xinjiang Medical University(20160114-12), and all participants signed informed consent forms.

\section{Acknowledgements}

We are extremely grateful to Dr. Tieming Jiang for the advice on the study design.

\section{Fundings}

This work was supported by grants from the National Natural Science Foundation of China (81860361, 81660341, 81760369), China Postdoctoral Science Foundation (2019M653802), Natural Science Foundation of Xinjiang Uygur Autonomous Region (2018D01C199), State Key Laboratory of Pathogenesis, Prevention and Treatment of Central Asian High Incidence Diseases Fund (SKL-HIDCA2019-30, SKL-HIDCA-2018-17, SKL-HIDCA-2019-22,SKL-HIDCA-2019-28).

\section{Author Contributions}

Renyong Lin, Guodong Lü and Ying Ke conceived and designed the study.

Ying Ke, Xiaojuan Bi, Ning Yang, Xiaohong li, Hui wang, Hui Liu and Liang Li performed the experiments. Wenmei Ma, Yongde Qin, Tuerganaili Aji, Yingmei Shao contributed to technical or materials related issues or clinical data collection.Ying Ke, Xiaojuan Bi and Cheng Li analyzed the data.Ying Ke, Xiaojuan $\mathrm{Bi}$ and Renyong Lin drafted the manuscript.Renyong Lin critically revised manuscript. Renyong Lin and Guodong Lü supervised the study.

All authors reviewed the manuscript.

\section{Consent for publication}

Not applicable.

\section{Competing Interests}

The authors have no conflicts of interest to disclose.

\section{References}

1. Kern P, Menezes da Silva A, Akhan O, Mullhaupt B, Vizcaychipi KA, Budke C, Vuitton DA. The Echinococcoses: Diagnosis, Clinical Management and Burden of Disease. Adv Parasitol 2017;96:259-369.

2. Wen H, Vuitton L, Tuxun T, Li J, Vuitton DA, Zhang W, McManus DP. Echinococcosis: Advances in the 21st Century. Clin Microbiol Rev 2019;32. 
3. Tuxun T, Apaer S, Ma HZ, Zhao JM, Lin RY, Aji T, Shao YM, et al. Plasma IL-23 and IL-5 as surrogate markers of lesion metabolic activity in patients with hepatic alveolar echinococcosis. Sci Rep 2018;8:4417.

4. Qin Y, Li X, Zhang Q, Xie B, Ji X, Li Y, Yiblayan A, et al. Analysis of the clinical value of (18)F-FDG PET/CT in hepatic alveolar echinococcosis before and after autologous liver transplantation. Exp Ther Med 2016;11:43-48.

5. Chen $\mathrm{PH}$, Chen X, He X. Platelet-derived growth factors and their receptors: structural and functional perspectives. Biochim Biophys Acta 2013;1834:2176-2186.

6. Borkham-Kamphorst E, Weiskirchen R. The PDGF system and its antagonists in liver fibrosis. Cytokine Growth Factor Rev 2016;28:53-61.

7. Chauhan A, Adams DH, Watson SP, Lalor PF. Platelets: No longer bystanders in liver disease. Hepatology 2016;64:1774-1784.

8. Üçüncü M, Serilmez M, Sarı M, Bademler S, Karabulut S. The Diagnostic Significance of PDGF, EphA7, CCR5, and CCL5 Levels in Colorectal Cancer. Biomolecules 2019;9.

9. Farooqi AA, Siddik ZH. Platelet-derived growth factor (PDGF) signalling in cancer: rapidly emerging signalling landscape. Cell Biochem Funct 2015;33:257-265.

10. Kilvaer TK, Rakaee M, Hellevik T, Vik J, Petris L, Donnem T, Strell C, et al. Differential prognostic impact of platelet-derived growth factor receptor expression in NSCLC. Sci Rep 2019;9:10163.

11. Kuo TL, Cheng KH, Shan YS, Chen LT, Hung WC. $\beta$-catenin-activated autocrine PDGF/Src signaling is a therapeutic target in pancreatic cancer. Theranostics 2019;9:324-336.

12. Hayashi T, Yamashita T, Terashima T, Suda T, Okada H, Asahina Y, Hayashi T, et al. Serum cytokine profiles predict survival benefits in patients with advanced hepatocellular carcinoma treated with sorafenib: a retrospective cohort study. BMC Cancer 2017;17:870.

13. Aryal B, Yamakuchi M, Shimizu T, Kadono J, Furoi A, Gejima K, Komokata T, et al. Predictive Value of Diminished Serum PDGF-BB after Curative Resection of Hepatocellular Cancer. J Oncol 2019;2019:1925315.

14. Brunetti E, Kern P, Vuitton DA. Expert consensus for the diagnosis and treatment of cystic and alveolar echinococcosis in humans. Acta Trop 2010;114:1-16.

15. Li J, Dong J, Yang L, Li X, Song T. Comparison of [(18)F]Fluorodeoxyglucose Positron Emission Tomography and Contrast-Enhanced Ultrasound for Evaluation of Hepatic Alveolar Echinococcosis Activity. Ultrasound Med Biol 2018;44:2199-2208.

16. Zhang C, Lin R, Li Z, Yang S, Bi X, Wang H, Aini A, et al. Immune Exhaustion of T Cells in Alveolar Echinococcosis Patients and Its Reversal by Blocking Checkpoint Receptor TIGIT in a Murine Model. Hepatology 2020;71:1297-1315.

17. Hazra A, Gogtay N. Biostatistics Series Module 6: Correlation and Linear Regression. Indian J Dermatol 2016;61:593-601. 
18. Panda SK, Jana PK. A multi-objective task scheduling algorithm for heterogeneous multi-cloud environment. In: 2015 International Conference on Electronic Design, Computer Networks \& Automated Verification (EDCAV); 2015. p. 82-87.

19. Ammann RW, Renner EC, Gottstein B, Grimm F, Eckert J, Renner EL. Immunosurveillance of alveolar echinococcosis by specific humoral and cellular immune tests: long-term analysis of the Swiss chemotherapy trial (1976-2001). J Hepatol 2004;41:551-559.

20. Chen CF, Feng X, Liao HY, Jin WJ, Zhang J, Wang Y, Gong LL, et al. Regulation of T cell proliferation by JMJD6 and PDGF-BB during chronic hepatitis B infection. Sci Rep 2014;4:6359.

21. Zhou J, Deng Y, Yan L, Zhao H, Wang G. Serum platelet-derived growth factor BB levels: a potential biomarker for the assessment of liver fibrosis in patients with chronic hepatitis B. Int $\mathrm{J}$ Infect Dis 2016;49:94-99.

22. Kurokawa T, Ohkohchi N. Platelets in liver disease, cancer and regeneration. World J Gastroenterol 2017;23:3228-3239.

23. Slaba I, Wang J, Kolaczkowska E, McDonald B, Lee WY, Kubes P. Imaging the dynamic plateletneutrophil response in sterile liver injury and repair in mice. Hepatology 2015;62:1593-1605.

\section{Figures}
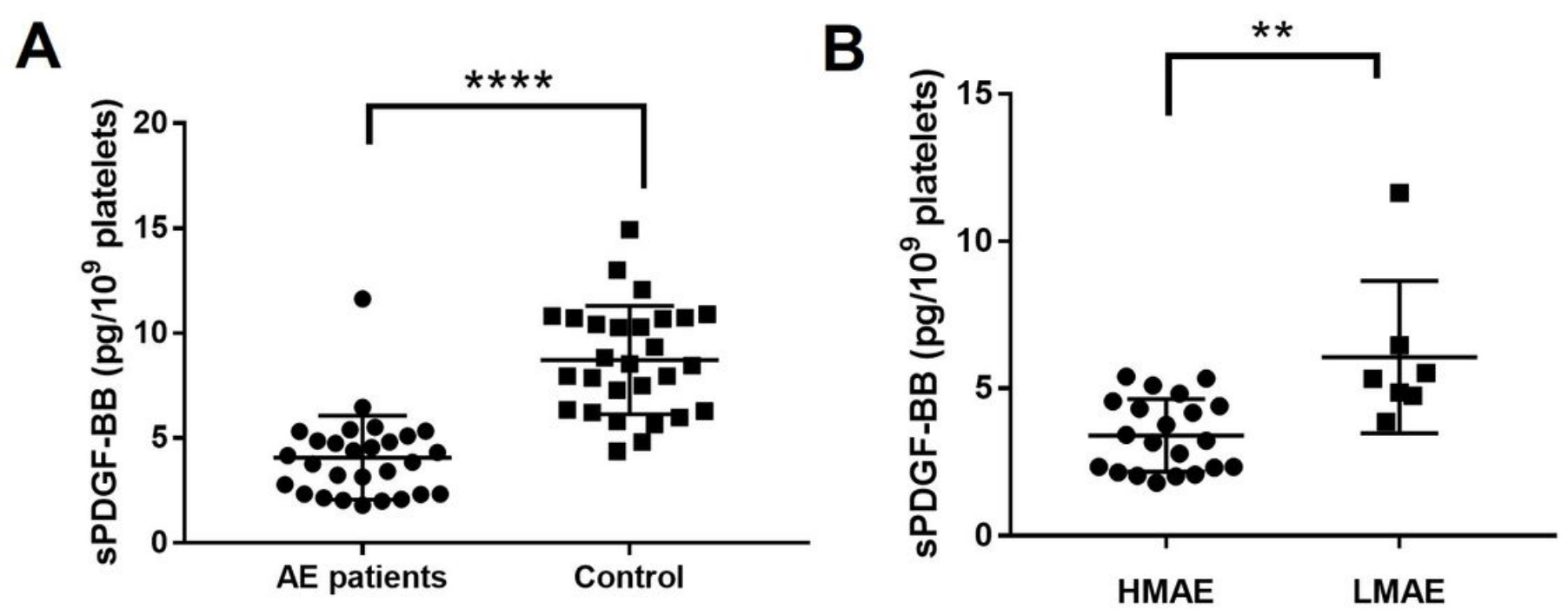

Figure 1

Low sPDGF-BB concentration in AE patients. (A) SPDGF-BB concentrations in AE patients $(n=28)$ and healthy control subjects $(n=28, * \star * * p<0.0001)$. (B) sPDGF-BB concentrations in HMAE $(n=21)$ and LMAE $(n=7, * \star p<0.01)$. 
A

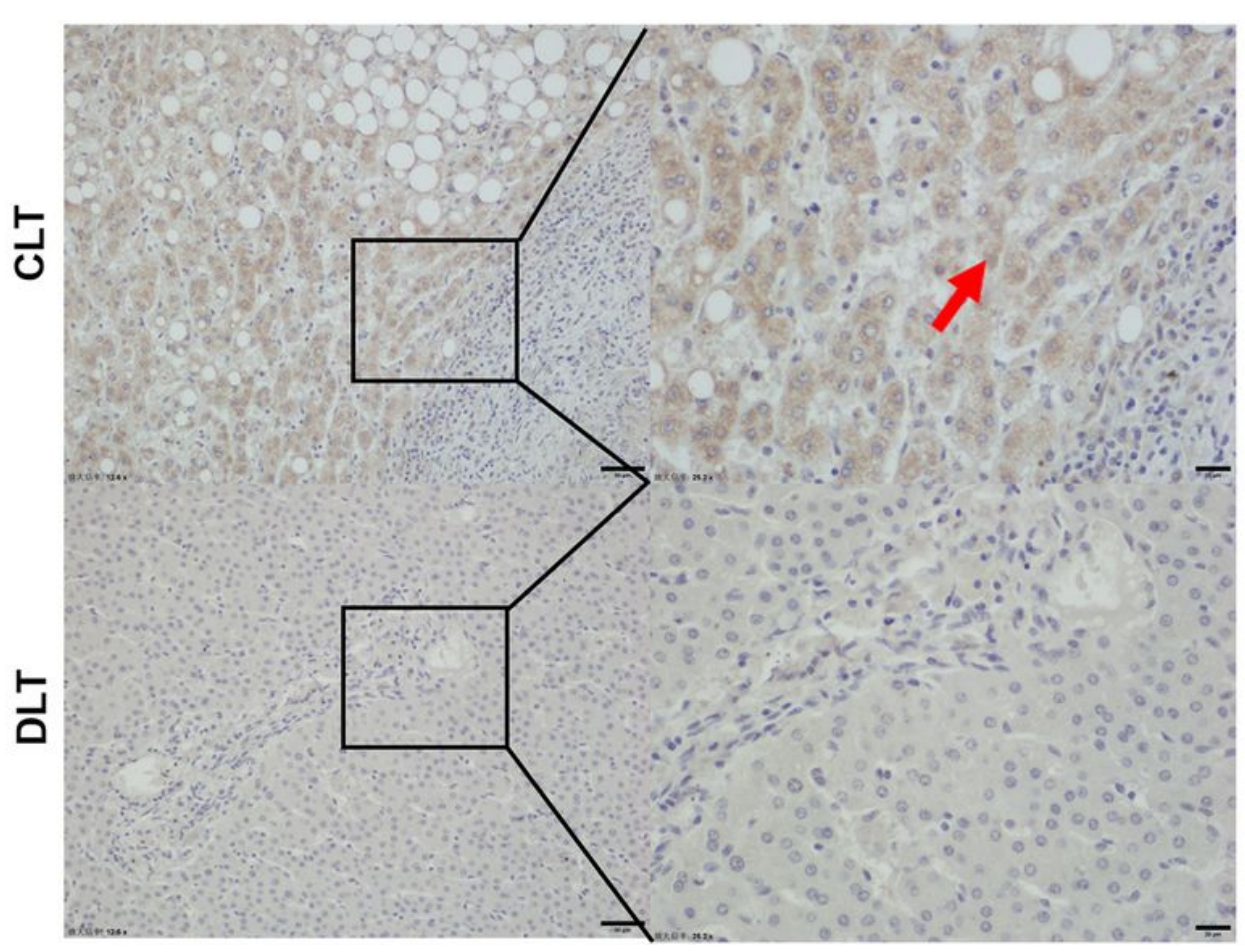

B

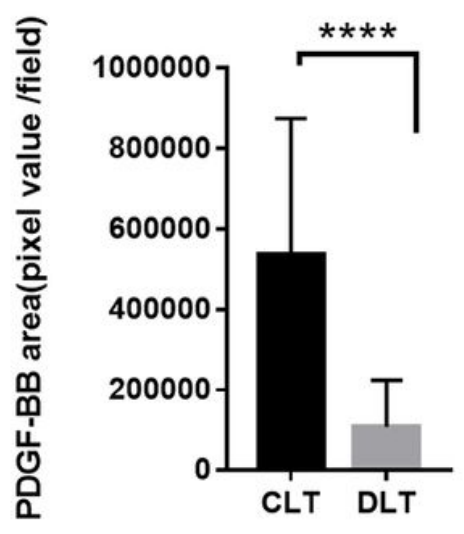

Figure 2

High expression of PDGF-BB in CLT from AE patients. (A) Representative immunohistochemistry staining for PDGF-BB in CLT from AE patients (left panel 100x, right panel 400x, arrowheads indicate PDGF-BB positive cells). (B) Quantitative analysis of the PDGF-BB expression in CLT region ( $n=28, * \star \star * p<0.0001)$.
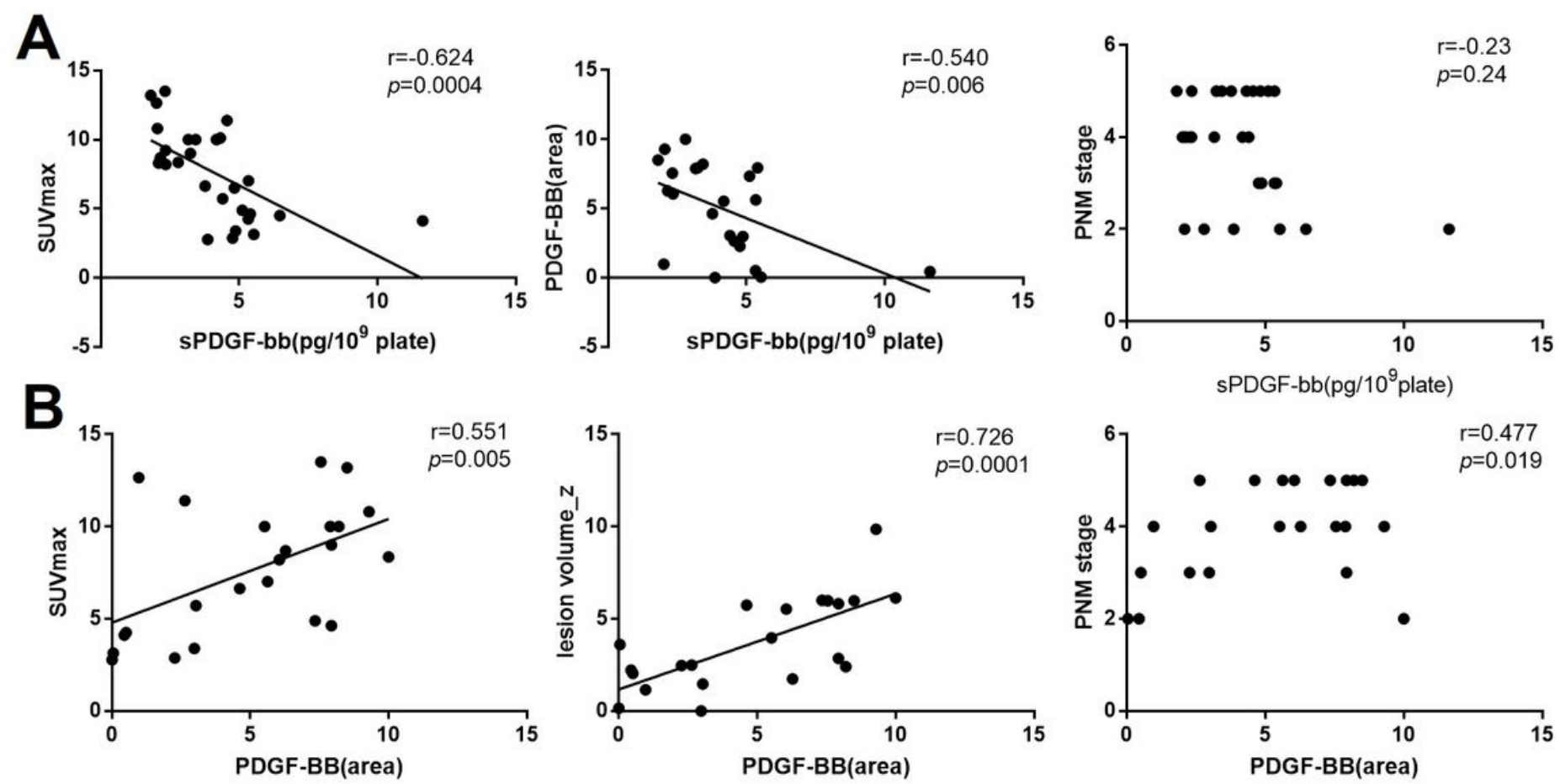

Figure 3 
Correlation analysis of sPDGF-BB/PDGF-BB expression with clinical variables in AE patients. (A) Correlation analysis of sPDGF-BB expression level with clinic variables of $A E$ patients. (B) Correlation analysis of PDGF-BB expression level in CLT with clinic variables of AE patients.

\section{Supplementary Files}

This is a list of supplementary files associated with this preprint. Click to download.

- FigureS2.jpg

- Figures1.tif

- supplementaryfigurelegends.docx

- TableS1.docx

- Tables2.xlsx 